\title{
Tumour heterogeneity: a problem in biopsy assessment of the proliferation index of oesophageal adenocarcinomas
}

\author{
K Jenner, S J Darnton, L Billingham, A T Warfield, H R Matthews
}

\begin{abstract}
Tumour heterogeneity may pose a problem when biopsy specimens are taken to measure proliferation (for example, in assessing response to therapy). Two "biopsy specimens" were taken from the centre and two from the edge of the luminal surface of 20 resected oesophageal adenocarcinomas. The proliferation index for each "biopsy specimen" was measured by counting Ki67 labelled nuclei in histological sections. The proliferation index was not associated with tumour differentiation or stage. There was site specific heterogeneity with a significant difference in proliferation index between the central (mean (SD) 36.4 (9.7)) and edge "biopsy specimens" (39.3 (9.9)). There was, however, a wide range of differences between pairs of "biopsy specimens" from both sites. In conclusion, if a tumour is to be sampled for measurement of the proliferation index before and after treatment, then the sequential biopsy specimens (preferably duplicated on each occasion) should be taken consistently from a leading edge of the lesion.

(f Clin Pathol: Mol Pathol 1996;49:M61-M63)
\end{abstract}

Department of

Thoracic Surgery,

Birmingham

Heartlands Hospital,

Birmingham B9 5SS

K Jenner

S J Darnton

H R Matthews

Department of

Histopathology

A T Warfield

The CRC Institute for Cancer Studies, The University of Birmingham School of Medicine, Birmingham B15 2TH

L Billingham

Correspondence to: Miss K Jenner,

Oesophageal Research Laboratory, Department of Thoracic Surgery, Birmingham Heartlands Hospital, Birmingham Bo 5SS.

Accepted for publication 12 October 1995
Keywords: carcinoma, heterogeneity, Ki67, oesophageal adenocarcinoma, proliferation.

Tumour growth is determined by the balance between cell gain (by proliferation) and cell loss (by apoptosis and necrosis). The former has been studied far more extensively than the latter. The proliferative level can be estimated histologically by several methods, of which the staining and counting of Ki67 labelled nuclei is considered to be the most simple and reliable. The Ki67 antibody recognises a nuclear protein in cells in all phases of the cell cycle except $\mathrm{G}_{0}{ }^{1}$ Counting the percentage of labelled cells within a tumour gives a proliferation index.

Adenocarcinoma of the oesophagus is an aggressive tumour with a poor prognosis (overall five year survival is less than $1 \%) .{ }^{2}$ Most patients have disseminated disease at presentation. Chemotherapy potentially has a role to play in the control of the disease. In a preliminary study using biopsy assessment of oesophageal squamous carcinomas, before and shortly after chemotherapy, results indicated that changes in Ki67 counts could be an indicator of response to treatment. ${ }^{3}$

Biopsy assessment, with concomitant sampling error, raises the problem of tumour heterogeneity with respect to the parameter to be measured. "To regard normal or pathological tissues as kinetically homogenous is a recipe for disaster!" (Ansari and Hall, 1992). ${ }^{4}$

In this study we have addressed the possible effect of tumour heterogeneity on the proliferation index of oesophageal adenocarcinomas by assessing multiple "biopsy specimens" taken from mapped sites on the surface of resected tumours.

\section{Methods}

Between 1990 and 1992, 20 patients (16 men, mean age 64 years, range 38-76 years) with oesophageal adenocarcinomas were studied prospectively. Patients received no neoadjuvant treatment. Following resection, the specimen was opened longitudinally and four mapped samples were taken immediately, using a fresh blade, from the luminal surface of the tumour: two from the central area and two from the growing edge. The samples were taken deliberately to resemble in size biopsy specimens taken at in vivo endoscopy (using a fixed endoscope, which is the normal practice in our Department of Thoracic Surgery). The dimensions were approximately $3 \mathrm{~mm} \times 2 \mathrm{~mm} \times 1 \mathrm{~mm}$. These were fixed for 24 hours in $10 \%$ formalin and processed routinely to paraffin wax.

Sections $(3 \mu \mathrm{m}$ thick) for immunohistochemistry were mounted on coated slides (Vectabond, Vector Laboratories, Peterborough, UK) and dried overnight at $37^{\circ} \mathrm{C}$. After rehydration, antigen retrieval involved placing slides in $0.01 \mathrm{M}$ citrate buffer $(\mathrm{pH} 6)$ in a microwave oven $(650 \mathrm{~W})$ for 15 minutes. Peroxidase activity was blocked with $0.6 \%$ hydrogen peroxidase in methanol for 15 minutes. The Vectastain Elite ABC Kit (Vector Laboratories) was used. The primary antibody, a mouse anti-human Ki67 monoclonal (Dako, High Wycombe, UK) diluted 1 in 20 in phosphate buffered saline (PBS), $\mathrm{pH} 7 \cdot 4$, was applied and the slides were incubated for 30 
Table 1 Proliferation indexes (\%) counted by two observers on two centre "biopsy specimens" and two edge "biopsy specimens" for each tumour

\begin{tabular}{|c|c|c|c|c|c|c|c|c|}
\hline \multirow[b]{2}{*}{ Patient no. } & \multicolumn{2}{|l|}{ Centre 1} & \multicolumn{2}{|l|}{ Centre 2} & \multicolumn{2}{|l|}{ Edge 1} & \multicolumn{2}{|l|}{ Edge 2} \\
\hline & Observer 1 & Observer 2 & Observer 1 & Observer 2 & Observer 1 & Observer 2 & Observer 1 & Observer 2 \\
\hline 1 & 27 & 27 & 32 & 33 & 23 & 18 & 31 & 36 \\
\hline 2 & 41 & 40 & 38 & 36 & 42 & 46 & 31 & 36 \\
\hline 3 & 54 & 55 & 35 & 36 & 52 & 48 & 39 & 40 \\
\hline 4 & 49 & 49 & 49 & 54 & 49 & 51 & 55 & 54 \\
\hline 5 & 19 & 24 & 36 & 37 & 30 & 28 & 50 & 46 \\
\hline 6 & 28 & 33 & 16 & 20 & 37 & 41 & 26 & 23 \\
\hline 7 & 44 & 39 & 39 & 36 & 40 & 45 & 45 & 48 \\
\hline 8 & 31 & 34 & 42 & 40 & 61 & 56 & 46 & 42 \\
\hline 9 & 39 & 35 & 44 & 39 & 56 & 46 & 46 & 42 \\
\hline 10 & 33 & 29 & 29 & 32 & 40 & 39 & 42 & 38 \\
\hline 11 & 35 & 38 & 29 & 29 & 25 & 22 & 28 & 33 \\
\hline 12 & 53 & 51 & 43 & 42 & 38 & 36 & 41 & 36 \\
\hline 13 & 26 & 28 & 23 & 26 & 36 & 34 & 30 & 33 \\
\hline 14 & 45 & 42 & 36 & 39 & 43 & 42 & 33 & 37 \\
\hline 15 & 25 & 30 & 35 & 32 & 38 & 40 & 32 & 37 \\
\hline 16 & 36 & 36 & 32 & 37 & 35 & 29 & 39 & 33 \\
\hline 17 & 59 & 59 & 34 & 45 & 46 & 53 & 53 & 53 \\
\hline 18 & 34 & 33 & 18 & 23 & 25 & 28 & 24 & 26 \\
\hline 19 & 25 & 22 & 32 & 27 & 22 & 24 & 41 & 36 \\
\hline 20 & 44 & 48 & 50 & 55 & 53 & 58 & 49 & 58 \\
\hline
\end{tabular}

minutes at room temperature. The chromogen (brown staining) was 3,3' diaminobenzidine tetrahydrochloride (DAB Peroxidase Substrate Kit, Vector Laboratories) with a light haematoxylin counterstain. The positive control was a tonsil sample. Negative controls were tonsil sections incubated in PBS and test slides incubated with non-specific mouse IgG1 (isotype matched) antibody (Dako) in place of Ki67 antibody and run in parallel.

Positively stained Ki67 nuclei were counted at a magnification of $\times 400$ (blind as to source), by the method of Rüschoff $e t a l,{ }^{5}$ to a cumulative mean. The result was expressed as a percentage (proliferation index). Two observers counted between 500 and 1500 nuclei per biopsy. If values from the two observers were not within $5 \%$ at the first count, a further blind count by both observers was made. Nuclei in necrotic areas of tumour were not counted.

Serial sections, $3 \mu \mathrm{m}$ thick, were stained routinely with haematoxylin and eosin and reported for stage and grade (according to the most poorly differentiated area) by the criteria of the UICC. ${ }^{6}$

\section{STATISTICS}

The data were tested for normality using the Shapiro-Wilk test and were shown to be normally distributed.

A parametric analysis of variance was carried out using a mixed model to investigate both inter-observer variability and the variability between "biopsy specimens" from each patient. This analysis was repeated for each site (centre and edge) separately to investigate further the variability between "biopsy specimens" from each patient.

The counts from the two observers were combined into an average for each "biopsy specimen" and then a two-way parametric anal-

Table 2 Statistical analysis of intra-site variability of proliferation indexes

\begin{tabular}{lllll}
\hline $\begin{array}{l}\text { Biopsy } \\
\text { sample type }\end{array}$ & $\begin{array}{l}\text { Difference } \\
\text { between means }\end{array}$ & $\begin{array}{l}\text { Standard } \\
\text { deviation }\end{array}$ & $\begin{array}{l}\text { Actual } \\
\text { range }\end{array}$ & $\begin{array}{l}\text { Expected range in which } \\
95 \% \text { of differences fall }\end{array}$ \\
\hline Central & $2 \cdot 2$ & $9 \cdot 3$ & -15 to $19 \cdot 5$ & $-16 \cdot 1$ to $20 \cdot 5$ \\
Edge & $0 \cdot 2$ & $9 \cdot 4$ & -19 to 14.5 & $-18 \cdot 2$ to $18 \cdot 6$ \\
\hline
\end{tabular}

ysis of variance was used to investigate intersite variability.

Analysis of any association between proliferation index and pathological factors was relatively crude. Each patient's proliferation index was taken as the mean of the eight separate values. A Wilcoxon two-sample test was used to test any association between differentiation and proliferation index (skewed data). Association with stage was tested by a twosample $t$ test (normally distributed data).

\section{Results}

The microwave antigen retrieval method gave strong positive nuclear staining with occasional cytoplasmic staining (ignored in counting). Normal stratified squamous epithelium showed positive internal control staining of the nuclei of parabasal cells.

Negative controls always gave negative nuclear staining. Counts of proliferation indexes for all samples are shown in table 1. The observers, one experienced (SJD) and the other inexperienced $(\mathrm{KJ})$ at counting, reached agreement to within $5 \%$ in $38(47.5 \%)$ of 80 samples after the first round of counting, with 32 of 42 of the remaining samples resolved after the second count. Of the 10 samples unresolved after the second count, only six were left unreconciled after a third count as $>5 \%$ different from each other (as both counters were individually consistent for each sample). Eleven tumours were stage IIA and nine were stage III; four tumours were grade 2 and 16 were grade 3 .

\section{STATISTICS}

There was no significant inter-observer variability $(p=0.41)$, but the variability between the four "biopsy specimens" from each patient was highly significant $(p=0.0001)$. This could have been accounted for by differences between sites (centre and edge). However, repeating the analysis of variance for central and edge "biopsy specimens" separately also gave a highly significant difference ( $p=0.0001$ in both cases). 
Further investigation into intra-site variability (between pairs of "biopsy specimens" for both centre and edge) revealed that for both types of specimen, the mean difference between pairs was small but the standard deviation was large (table 2). This showed that there was a large amount of variation in the differences. This was also shown by the wide range of differences observed at each site, with differences as large as 19.5 being observed for the centre and 19 for the edge. Table 2 also shows, for pairs of "biopsy specimens", the range of values in which one would expect $95 \%$ of differences to occur.

There was significant inter-site variability $(\mathrm{p}=0 \cdot 04)$, with the central "biopsy specimens" having a mean (SD) of $36 \cdot 4(9 \cdot 7)$ and edge "biopsy specimens" having a mean of $39 \cdot 3$ (9.9).

The proliferation index did not seem to be associated with either differentiation $(p=0.99)$ or stage $(p=0.57)$.

\section{Discussion}

In some tumours the $\mathrm{Ki} 67$ proliferative index has been shown to correlate with stage and prognosis-for example, in lymphomas and breast carcinomas. ${ }^{78}$ In the present study the Ki67 index in oesophageal adenocarcinomas did not correlate with pathological stage or grade (although numbers were small). These results concur with those of Porschen et al, ${ }^{9}$ who found no correlation between pathological variables and the Ki67 index in tumours of the alimentary canal, including oesophageal squamous cell carcinomas.

Immunostaining was carried out on paraffin wax embedded tissue using the microwave antigen retrieval method. This obviates the use of frozen sections. Microwave retrieval gave reliable, strong, easily counted, nuclear staining. The running mean counting method was used to eliminate intra-sample heterogeneity. Accuracy was improved on repeated counts by consensus agreement as to the normal, dysplastic and neoplastic areas within difficult "biopsy specimens". Diffusely infiltrating, poorly differentiated tumours might have presented a particular problem in the distinction of neoplastic from stromal cells. With the close counting system, it was not surprising that statistical analysis of the data revealed no significant interobserver variability $(p=0.41)$.

There was a highly significant difference in the proliferation indexes from the two sites, centre and edge (means of 36.4 and 39.3 , respectively). The greater proliferation level of the edge could be related to a number of factors, consequent on the relation of the edge position to vascular supply.

Pathologists have assumed that the leading edge of the tumour is the most biologically active $^{10}$ and the present study supports this hypothesis, at least for oesophageal adenocarcinomas.

It follows, from these observations, that if one is taking sequential biopsy specimens in order to assess proliferation of a tumour, one should sample consistently from either the centre or the edge. Probably the latter is to be preferred, as problems consequent on necrosis in central ulcerated regions would thus be avoided. However, the analysis of intra-site variability within either the centre or edge site of a single tumour showed that there was a wide range of differences in indexes at both sites. When working with a tumour from a different site, investigators might be advised to make a preparatory study of heterogeneity.

In conclusion, we recommend that sequential biopsy specimens should be taken consistently from an edge site if proliferation is to be measured as a possible indication of response to treatment. Multiple biopsy specimens should also be obtained assuming that clinical considerations permit.

$\mathrm{KJ}$ and SJD are supported by the Oesophageal Cancer Research Appeal (OCRA), Birmingham.

1 Gerdes J, Lemke H, Baisch H, Wacker H-H, Schwab U, Stein $\mathrm{H}$. Cell cycle analysis of a cell proliferation-associated human nuclear antigen defined by the monoclonal antibody Ki-67. F Immunol 1984;133:1710-15.

2 Matthews HR, Waterhouse JAH, Powell J, Robertson JE, McConkey CC (eds). Overall Survival. In: Clinical cancer monographs. Vol 1. Cancer of the oesophagus. London: Macmillan, 1987:59-91.

3 Darnton SJ, Korkolopoulou P, Jenner K, Crocker J, Matthews HR. Apoptosis and expression of p53 and $\mathrm{Ki}-67$ in squamous carcinoma of the oesophagus before and shortly after treatment with systemic chemotherapy [abstract]. $\mathcal{F}$ after treatment with systemic
Pathol 1993;170(Suppl):362A.

4 Ansari B, Hall PA. The kinetic organisation of tissues. In: Hall PA, Levison DA, Wright NA, eds. Assessment of cell proliferation in clinical practice. London: Springer-Verlag, 1992:45-61.

5 Rüschoff J, Plate $\mathrm{KH}$, Contractor H, Kern S, Zimmermann $\mathrm{R}$, Thomas C. Evaluation of nucleolus organizer regions (NORs) by automatic image analysis: A contribution to standardization. F Pathol 1990;161:113-18.

6 Hermanek P, Sobin LH (eds). UICC International Union against Cancer. TNM classification of malignant tumours.
4th edn. 2nd revision. Berlin: Springer Verlag, 1992:42-4.

7 Hall PA, Levison DA. Assessment of cell proliferation in histological material [review]. F Clin Pathol 1990;43:18492

8 Yu CC-W, Woods AL, Levison DA. The application of immunohistochemistry in assessment of cellular proliferation. In: Hall PA, Levison DA, Wright NA, eds. Assessment of cell proliferation in clinical practice. London: Springer-Verlag, 1992:141-59.

9 Porschen R, Kriegel A, Langen C, Classen S, Hilse M, Lohe B, et al. Assessment of proliferative activity in carcinomas of the human alimentary tract by $\mathrm{Ki}-67 \mathrm{im}$ munostaining. Int $\mathcal{f}$ Cancer 1991;47:686-91.

10 Barnes DM, Gillet CE. Determination of cell proliferation. 7 Clin Pathol: Mol Pathol 1995;48:M2-5. 